\title{
Length-dependent toxicity of untranslated CUG repeats on Caenorhabditis elegans
}

\author{
Kuan-Yu Chen ${ }^{\text {a,b }}$, Huichin Pan ${ }^{\mathrm{b}}$, Min-Jon Lin ${ }^{\mathrm{b}}$, Yet-Young Li ${ }^{\mathrm{a}}$, Li-Chun Wang ${ }^{\mathrm{b}}$, \\ Yi-Chun $\mathrm{Wu}^{\mathrm{c}}$, Kuang-Ming Hsiao ${ }^{\mathrm{b}, *}$ \\ a Institute of Medicine, Chung Shan Medical University, Taichung 402, Taiwan, ROC \\ ${ }^{\mathrm{b}}$ Department of Biomedical Sciences, Chung Shan Medical University, Taichung 402, Taiwan, ROC \\ ${ }^{\mathrm{c}}$ Institute of Molecular and Cellular Biology, National Taiwan University, Taipei 10617, Taiwan, ROC
}

Received 9 November 2006

Available online 27 November 2006

\begin{abstract}
Expansion of CTG repeat within the $3^{\prime}$-untranslated region of the DMPK gene causes the most common neuromuscular disorder, myotonic dystrophy type 1 (DM1), through a RNA trans-dominant mechanism. Here, we explore Caenorhabditis elegans as a model system to investigate the repeat size-dependent toxic effect by expression of green fluorescent protein $(G F P)$ transcripts with various lengths of untranslatable CUG repeats (CUG5, CUG30, CUG83, CUG125, and CUG213) in body wall muscles. CUG213 animals died during embryogenesis or showed retarded growth at larval stages due to defective muscle development. CUG125 animals, although can produce offspring, exhibited uncoordinated muscle function, deviated electropharyngeogram, and an age-dependent abnormality in muscle structure. Most CUG83 animals had normal muscle structure and function as those expressing 30 and shorter repeats. Our results demonstrate for the first time that the in vivo toxicity of CUG repeats is repeat length- and growth-regulated and suggest that expanded CUG repeats are sufficient to cause congenital-like phenotypes in living organisms.
\end{abstract}

(C) 2006 Elsevier Inc. All rights reserved.

Keywords: DM1; CUG repeats; Transgenic C. elegans

Myotonic dystrophy 1 (DM1) is a dominantly inherited neuromuscular disorder caused by an expansion of CTG repeats in the $3^{\prime}$-untranslated region (UTR) of the dystrophia myotonica protein kinase $(D M P K)$ gene [1]. In normal population, the repeat number ranges in size from 5 to 37. Mild DM1 patients typically have fewer than 100 repeats and only present with cataracts. Classical DM1 patients usually have more than 100 repeats with an onset age around adolescence and develop multiple symptoms, including myotonia, progressive muscle weakness and atrophy, cardiac conduction defects, insulin intolerance, infertility, and reduced life expectancy. Congenitally affected children typically have more than 1000 CTG repeats and

\footnotetext{
* Corresponding author. Fax: +886 423248109.

E-mail address: kmh@csmu.edu.tw (K.-M. Hsiao).
}

are characterized by hypotonia and mental retardation. In addition to DM1 mutation, patients with a CCTG repeat expansion in intron 1 of zinc-finger protein 9 (ZNF9) gene (DM2) [2] also develop typical clinical manifestations of DM1 with the exception of a congenital form [3].

The findings that both transcribed but not translated CTG and CCTG repeats causing similar phenotypes indicate a toxic gain of function of the expanded repeats RNA. Consistently, mice expressing expanded CUG repeat in an unrelated transcript develop typical features of DM phenotypes [4]. However, unlike the pathological effects of expanded CUG repeat RNA on mammals, the untranslated $(\mathrm{CUG})_{162}$ repeats do not cause any toxicity in Drosophila [5]. Previously, the full length human SCA8 transcripts containing an untranslated $(\mathrm{CUG})_{9}$ or $(\mathrm{CUG})_{112}$ repeat tract both were shown to cause similar 
neurological phenotypes in Drosophila [6], suggesting that the expanded CUG repeats RNA is not the major pathological agent. These observations reveal that various model organisms may have a differential response to the expanded CUG repeat RNA.

The nematode Caenorhabditis elegans has been used to address the underlying pathogenic mechanisms of several neurological malfunctions, including polyglutamine (polyQ) disorders [7-10]. To investigate if the expanded CUG repeats-mediated pathogenic mechanism is also present in organisms other than mammals and to further clarify the correlation between the repeat sizes and their physiological effect, we have here generated transgenic $C$. elegans expressing various lengths of CTG repeats in the $3^{\prime}$-UTR of a reporter gene in body wall muscles. Our results demonstrate for the first time that CUG repeats are toxic to $C$. elegans in a length- and growth-dependent manner. Furthermore, they indicate that expanded CUG repeats alone are sufficient to cause severe developmental defects observed in congenital cases.

\section{Materials and methods}

Plasmid constructs. pPD118.20 vector was used as a control plasmid in which the GFP gene was driven by the myo3 promoter. The plasmid myo3::g $f p(C T G)_{5}$ was constructed by annealing equal amounts of two synthetic oligonucleotides, $5^{\prime}-\mathrm{CCGGA}(\mathrm{CTG})_{5} \mathrm{~T}-3^{\prime}$ and $5^{\prime}-\mathrm{CCGGA}(-$ $\mathrm{CAG})_{5} \mathrm{~T}-3^{\prime}$, and then inserting into the BspEI site of pPD118.20 vector. The other constructs with longer CTG repeats were obtained by PCR using $(\mathrm{CTG})_{10}$ and $(\mathrm{CAG})_{17}$ as primers [11]. Successful construction of plasmids was confirmed by DNA sequencing.

Caenorhabditis elegans protocols. Transgenic animals were produced by standard transformation techniques [12] and the animals were grown and maintained following standard methods [13]. For CUG213 animals, morphological examination of $\mathrm{F} 1$ dead embryos and growth-retarded larva was observed using a $40 \times$ objective on a Zeiss Axioskop 2 microscope. Preparation of synchronized cultures and specific stages of animals were performed by using alkaline hypochlorite method [14].

Fluorescence microscopy. To examine the GFP fluorescence, individual worms were put on a fresh plate at day 2 after hatching and were observed at days 2-4 (equals to L2 to adult stage) on a Zeiss Stemi SV11 dissection microscope using a $6.6 \times$ objective and photographs were taken using Nikon coolpix 4500 digital camera.

Western blot analysis. Twenty micrograms of protein prepared from mixed populations of transgenic worms was resolved by SDS/PAGE, transferred onto PVDF membrane (NEN ${ }^{\top M}$ Life Science Products), and immunoblotted with the anti-GFP antibody (Santa Cruz Biotechnology) at a dilution of 1:1500 and the anti-actin antibody (MD Bio, Taiwan) at a dilution of 1:1000. Horseradish peroxidase (HRP)-conjugated Anti-Mouse IgG (Santa Cruz Biotechnology) was used at a dilution of 1:3000. Visualization was performed using a chemiluminescent detection kit (Pierce Chemical Company).

Quantitative real-time $R T$-PCR. Total RNA was extracted from young adult worms using TRI reagent (Molecular Research Center, Inc.). Oligo(dT) primed first strand cDNA was synthesized from $2 \mu \mathrm{g}$ of total RNA using the SuperScript kit (Invitrogen). Quantitative RT-PCR was performed using the SYBR Green double stranded DNA binding dye (Perkin-Elmer Life Sciences). The GFP transcripts were amplified using primers 5'-TTTCACTGGAGTTGTCCCAATTC-3' and 5'-CTTCACC CTCTCCACTGACA GAA- $3^{\prime}$. For controls, a C. elegans actin primer pair 5'-ATCGTCCTCGACTCTGGAGATG-3' and 5'-TCACGTCCA GCCAAGTCA AG-3' was used. Cycling parameters were $95^{\circ} \mathrm{C}$ for $10 \mathrm{~min}$, followed by 40 cycles of $95^{\circ} \mathrm{C}$ for $15 \mathrm{~s}$ and $60^{\circ} \mathrm{C}$ for $1 \mathrm{~min}$.
Following PCR amplification, $8 \mu \mathrm{l}$ of each PCR product was run on a $1.2 \%$ agarose gel.

Locomotory behavior. To examine locomotion, L4 worms were picked to fresh spread plates and their tracks were recorded after $24 \mathrm{~h}$ by using a Zeiss Stemi SV11 dissection microscope at magnification 20x.

Muscle morphology. To examine the musculature, phalloidin staining was performed as described in [15]. Photographs were taken on a Zeiss Axioplan fluorescence microscope using a 100× objective.

Electropharyngeogram (EPG). EPG analysis was performed by using extracellular recordings methods based on those of Raizen and Avery [16]. Adult worms were transferred into an approximately $1 \mathrm{~cm}$ diameter drop of Dent's saline solution on a $35 \times 50 \mathrm{~mm}$ glass coverslip. Dent's saline [17]. Electrophysiological preparations were observed under a Zeiss Axiovert 25 inverted microscope. The tip of the head was sealed by suction into fire-polished borosilicate glass electrodes of about $15-30 \mu \mathrm{m}$ outer diameter. Recording electrodes were filled with internal saline $(136.5 \mathrm{mM}$ potassium gluconate, $17.5 \mathrm{mM} \mathrm{KCl}, 9 \mathrm{mM} \mathrm{NaCl}, 1 \mathrm{mM} \mathrm{MgCl} 2,10 \mathrm{mM}$ Hepes, and 0.2 mM EGTA, pH 7.4). EPGs were recorded with an AxoClamp-2B microelectrode amplifier (Axon) connected to a Digidata 1322 A (Axon Instrument., USA) and a computer with pClamp 9.0 software. The analysis of electrophysiological signals is according to the previous interpretation [17].

\section{Results}

Expression of untranslated $(C U G)_{213}$ repeats causes severe developmental defects in $C$. elegans

To investigate if the untranslated CUG repeats are toxic to $C$. elegans, we have initially tried to generate transgenic C. elegans expressing GFP transcripts with 213 repeats of CUG triplets (CUG213). Most animals died within a few days after injection. A few of injected animals may lay eggs. However, these eggs stopped developing at three- to fourfold stage during embryogenesis or showed retarded growth at L2 stage with abnormal body shape as shown in Fig. 1. The expression of transgenes was confirmed by using fluorescent microscope. Compared to the control animals, the uneven distribution of GFP protein revealed by fluorescence images indicated that the muscle structure was severely disrupted in the defective embryos and larva

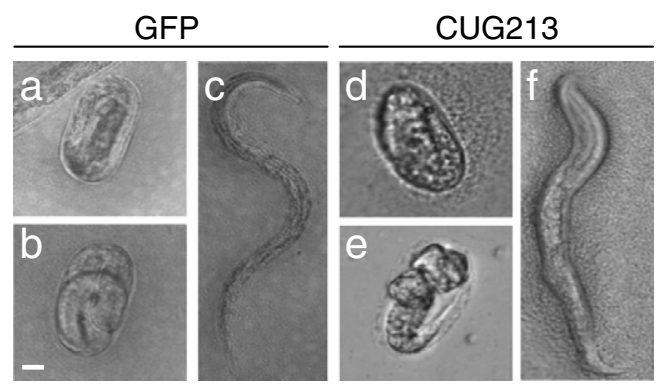

Fig. 1. Defective development of CUG213 transgenic C. elegans. (a-c) Representative Nomarski images of GFP animals at (a) the threefold stage, (b) the embryonic stage before hatching, and (c) the late L2 larval stage (approximate $22 \mathrm{~h}$ after hatching at $22{ }^{\circ} \mathrm{C}$ ). (d,e) CUG213 embryos at approximately the same stage as those of $(\mathrm{a}, \mathrm{b})$, respectively. They failed to elongate further and remained unhatched. The animal in (f) was CUG213 larva of $\sim 4$ day after hatching. Although it was able to feed, it remained paralyzed and had abnormal morphology. The scale bar represents $10 \mu \mathrm{m}$ and applies to all panels. 
(Supplementary Fig. 1). Obviously, the expression of GFP transcripts containing 213 repeats of CUG triplets was sufficient to cause lethal effects on this simple animal.

There is a toxic size threshold between 83 and 125 repeats of CUG triplets in C. elegans

Subsequently, transgenic C. elegans expressing 5, 30, 83, and 125 CUG repeats, designated as CUG5, CUG30, CUG83, and CUG125, were generated. Examination of the transgene expression by using fluorescent microscope revealed a repeat length-dependent decrease of fluorescence signal during development (Supplementary Fig. 2). The inverse correlation between repeat length and GFP protein level was confirmed by Western blotting (Fig. 2A). To investigate if the decrease of GFP protein level is due to the reduction of RNA expression, quantitative RT-PCR was performed using total RNA. All examined animals expressed similar levels of GFP RNA (Fig. 2B), indicating that large CUG repeats in the $3^{\prime}$-UTR did not interfere with gene transcription.

The muscle phenotypes of transgenic animals, including locomotory pattern and muscle structure, were analyzed. As expected, all GFP, CUG5, and CUG30 animals moved with a regular S pattern. There were some $(<10 \%)$ CUG83
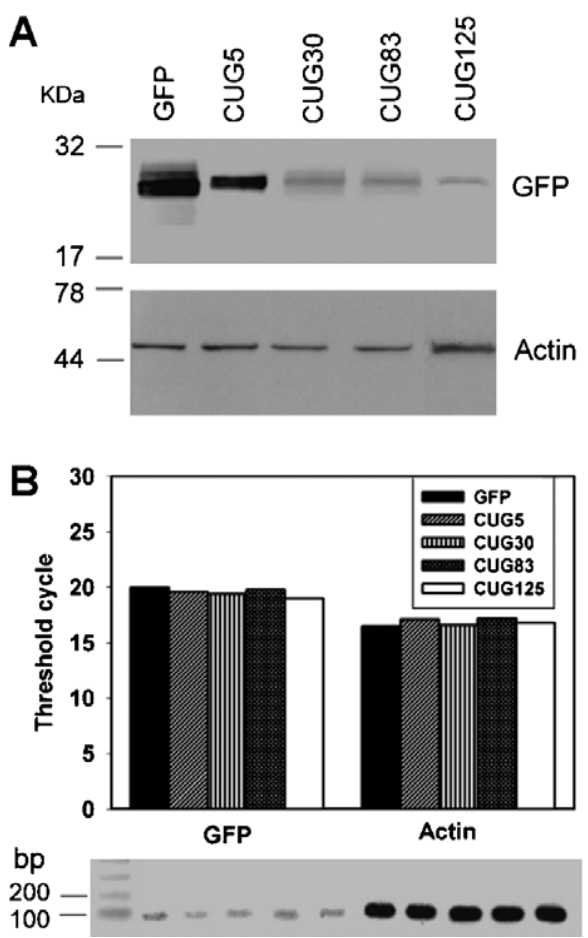

Fig. 2. The expression of transgenes in C. elegans. (A) Western blot analysis of GFP protein. (B) The expression level of GFP mRNA. The threshold cycle is shown in the upper panel. Confirmation of a single product of amplification in quantitative real-time RT-PCR is shown in the bottom panel. A 83-bp cDNA fragment was amplified from GFP RNA, and a 100-bp cDNA fragment was amplified from actin RNA. Size markers in bp are indicated. Actin mRNA and protein were used as an internal control in both experiments. adult animals showing irregular movement pattern occasionally. In contrast, most CUG125 adult animals frequently left abnormal, heavy tracks on the plate which indicated an impairment of locomotion (Fig. 3A). Further examination of the musculature by staining animals with the filamentous actin (F-actin) marker phalloidin revealed disrupted, wave-like structure in the body wall muscles (Fig. 3B). On average, there were 4-5 abnormal muscle cells observed in each young adult animal. One to two abnormal muscles can be detected in some CUG83 animals. No abnormal cell was detected in animals expressing shorter repeats. Altogether, these findings suggest a lengthdependent toxicity of untranslated CUG repeats to the muscle function and structure in $C$. elegans and indicate that this toxicity is from the CUG repeat RNA.

\section{The deleterious effect of untranslated CUG repeats on muscle structure is growth-dependent}

Most classical DM1 patients present clinical phenotypes around adolescence. Meanwhile, we have observed a progressively increasing ratio of CUG125 animals with uncoordinated muscle function during development (data not shown). To further confirm if the CUG repeats-mediated toxicity in C. elegans is growth-dependent, we examined the musculature of transgenic animals from L2 to adult. As expected, animals expressing 30 or less CUG repeats had a normal pattern of body wall muscles which consisted of a regular arrangement of spindle-shaped cells. Approximate $7 \%$ of CUG83 animals at L3 stage were found to have 1-2 abnormal muscle cells and the frequency increased to $10 \%$ in adults. A much more dramatic increase of the frequency of CUG125 animals with abnormal muscle cells was noticed at the L3-L4 transition (Fig. 3C). There were only $10 \%$ of L3 animals having abnormal muscle cells, but up to $60 \%$ of L4 animals and $75 \%$ of adults had disorganized body wall muscles. The increasing frequency of altered muscle F-actin pattern and defective locomotion behavior during development of CUG125 animals suggest that the deleterious effect of CUG repeats on muscles is growth-dependent.

\section{CUG125 transgenic worms show abnormal electropharyngeogram (EPG)}

In addition to the muscle phenotype, we also investigated if the expanded CUG repeats cause abnormal electrophysiological response by performing EPG analysis. The typical EPG consists of a contraction followed by a relaxation. The contraction potential, which is the large positive transient in the EPG, corresponds to the depolarization of pharyngeal muscle and the onset of contraction. The relaxation potential, which is the large negative transient, corresponds to the repolarization of pharyngeal muscle and the end of action potential. The amplitudes of positive and negative transient are above 2 and $3 \mathrm{mV}$, respectively, in all examined control animals. In contrast, they are below 
A
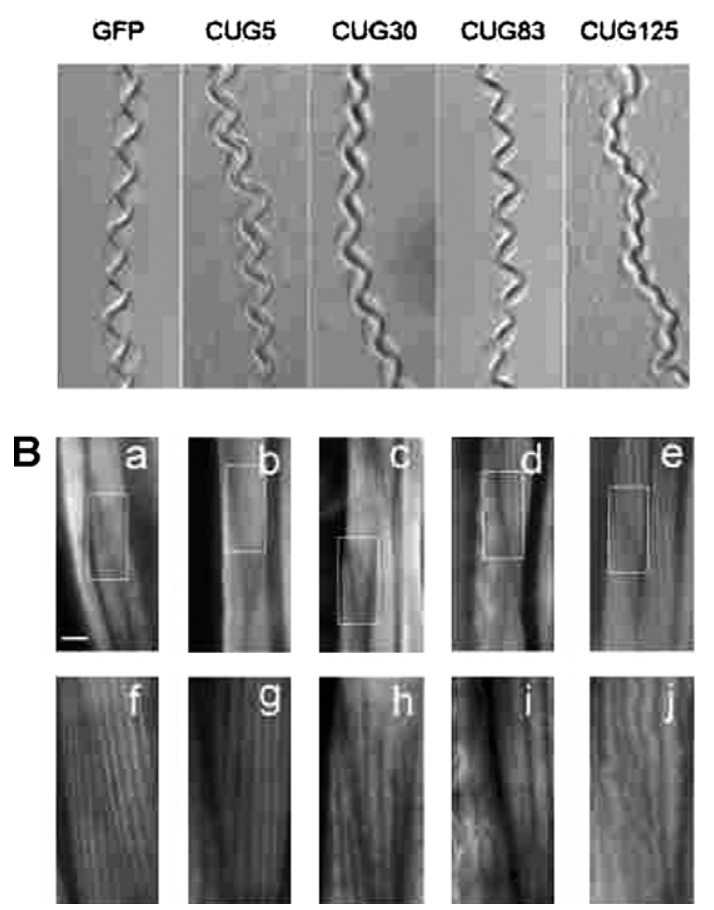

C

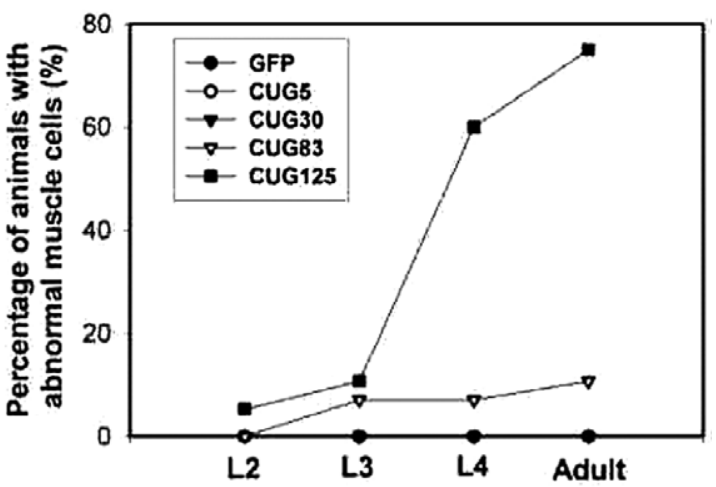

D

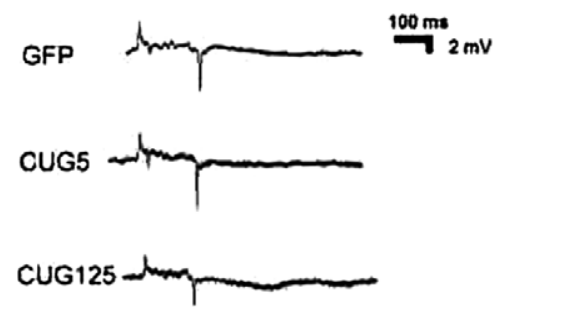

Fig. 3. The toxicity of long CUG repeats to C. elegans. (A) Locomotory patterns of transgenic animals expressing different lengths of CUG repeats. (B) Muscle structure of phalloidin-stained GFP (a), CUG5 (b), CUG30 (c), CUG83 (d), and CUG125 (e) animals. (f-j) Enlargement of areas delimited by white boxes in (a-e), respectively. The scale bar is $25 \mu \mathrm{m}$. (C) Growth-dependent effect of CUG repeats on muscle structure. Percentage represents the ratio of the animals with abnormal muscle cells to the total examined animals. At least 30 animals were examined for each data point. (D) Representative EPG recorded from GFP, CUG5, and CUG125 transgenic animals.

Table 1

Electropharyngeography analysis of transgenic worms

\begin{tabular}{lll}
\hline $\begin{array}{l}\text { Transgenic animals } \\
\text { (number of examined } \\
\text { animals) }\end{array}$ & $\begin{array}{l}\text { Contraction } \\
\text { potential } \\
\text { (means } \pm \mathrm{SE}, \mathrm{mV})\end{array}$ & $\begin{array}{l}\text { Relaxation potential } \\
\text { (means } \pm \mathrm{SE}, \mathrm{mV})\end{array}$ \\
\hline GFP $(6)$ & $2.4 \pm 0.2$ & $4.0 \pm 0.5$ \\
CUG5 (4) & $2.6 \pm 0.4$ & $3.7 \pm 0.6$ \\
CUG125-1 (10) & $1.5 \pm 0.3^{\mathrm{a}}$ & $2.6 \pm 0.3^{\mathrm{a}}$ \\
CUG125-2 (13) & $1.8 \pm 0.2$ & $2.9 \pm 0.2^{\mathrm{a}}$ \\
CUG125-3 (7) & $1.4 \pm 0.2^{\mathrm{a}}$ & $2.3 \pm 0.3^{\mathrm{a}}$ \\
\hline
\end{tabular}

${ }^{\text {a }}$ Statistically different from GFP animals using unpaired Student's test, $P<0.05$.

2 and $3 \mathrm{mV}$, respectively, in the majority of CUG125 animals (as shown in Fig. 3D). The positive and negative transient in three examined lines for CUG125 worms is shown in Table 1. Our results indicate that expanded CUG repeats may affect the electrophysiological behavior of $C$. elegans.

\section{Discussion}

In this study, we have investigated the toxic effects of untranslated CUG repeats using $C$. elegans as a model. Our results confirmed that CUG repeats are toxic in a length-dependent manner in this system. Moreover, we demonstrate for the first time that expanded CUG RNA repeats are sufficient to cause embryonic lethality in living organisms.
It was previously shown that mice expressing 250 or 300 CUG repeats in either unrelated or DMPK transcripts developed characteristic features of DM $[4,18]$. In contrast, RNA containing 162 CUG repeats did not cause any phenotypes in Drosophila [5]. A plausible explanation for the contradictory observations is that the pathological size threshold of CUG repeats is between 162 and 250. Therefore, we first tested the effect of expanded CUG in C. elegans using transgene containing 213 repeats. Unexpectedly, animals expressing GFP transcripts with this size of repeats either died during embryogenesis or were growth-arrested at the larva stages, presumably due to defects in muscle development (Fig. 1). It suggests that the congenital form-like phenotypes of DM, which has never been demonstrated by expressing CUG expansion alone in other animal models, can be reproduced in this C. elegans system.

The differential responses to expanded CUG repeats in these model organisms suggest that there may be distinct cell type- or species-specific factors that are important in mediating the toxic pathways. Thus, the Drosophila model may be suitable for observation of neurodegeneration $[6,19,20]$ but not for the DM muscle phenotype [5] due to the lack of specific mediating factors. Indeed, ELAV is a RNA-binding protein essential for neurogenesis in Drosophila and there are two C. elegans homologues of ELAV identified, ELAV-type RNA-binding protein (ETR-1) and ELAV-like protein (ELR-1). ETR-1 is a muscle-specific 
RNA-binding protein which shares up to $74 \%$ identity with human CUG RNA-binding protein (CUG-BP) within the RNA recognition motifs (RRMs) [21]. The human CUG$\mathrm{BP}$ has been implicated in the DM pathogenesis and ETR-1 has also been shown to play an essential role in muscle development [22]. However, there is only $28 \%$ identity over the same regions between ETR-1 and ELAV genes. ELR-1 is more closely related to Drosophila ELAV and they share 59\% identity in the RRMs [23]. ELR-1 and its closely related ELAV homologues in vertebrate family members share neuronal expression patterns that suggest a distinct tissue-specificity of their functions. Therefore, it is likely that Drosophila may lack the pathological mediator for expanded CUG repeats. Alternatively, the size threshold of toxicity for CUG repeats could vary in different species and this threshold may be over 162 in Drosophila. However, in C. elegans, 125 repeats are sufficient to exert detrimental effects on the muscle structure and function and on the electrophysiological behavior (Fig. 3). Further experiments using longer triplet repeats in Drosophila may address this hypothesis.

We noticed that worms expressing 83 CUG repeats had a slight toxicity on the muscles (Fig. 3B). Although no obvious effect on muscle function in most CUG83 animals was observed, it raises the possibility that CUG repeats of this size may cause a mild toxicity at later stages which are beyond the age of animals analyzed in this study. In human, benign DM1 patients having 50-100 CUG repeats present little neuromuscular abnormality during his life. Classical patients having more than 100 repeats usually have muscular and neurological phenotypes. This repeat size-phenotype relationship suggests that the threshold sizes of toxic CUG repeats are similar in C. elegans and human. Meanwhile, consistent with an onset age of around adolescence in classical patients, we have demonstrated here that the toxic effect of expanded CUG repeats is agedependent.

Since the CUG repeats cannot be translated, the phenotypes of CUG125 and CUG213 transgenic worms should be mediated by toxic repeat RNA. Indeed, the GFP animals were normal in all examined neuromuscular phenotypes and although the GFP protein expression levels were decreased by long repeats, the GFP RNA level remained unchanged (Fig. 2). Therefore, C. elegans is not only suitable for the mechanical and therapeutic studies of protein-mediated neurological disorders but also can be a good model system to investigate the effect of expanded repeats RNA.

\section{Acknowledgments}

This work was supported by grants from the National Science Council, Taiwan (NSC 92-2320-B-040-041, NSC 93-2320-B-040-063, NSC 94-2320-B-040-012, and NSC 95-2320-B-040-002) to K.M.H.

\section{Appendix A. Supplementary data}

Supplementary data associated with this article can be found, in the online version, at doi:10.1016/j.bbrc. 2006.11.102.

\section{References}

[1] M. Mahadevan, C. Tsilfidis, L. Sabourin, G. Shutler, C. Amemiya, G. Jansen, C. Neville, M. Narang, J. Barcelo, K. O’Hoy, et al., Myotonic dystrophy mutation: an unstable CTG repeat in the $3^{\prime}$ untranslated region of the gene, Science 255 (1992) 1253 1255 .

[2] C.L. Liquori, K. Ricker, M.L. Moseley, J.F. Jacobsen, W. Kress, S.L. Naylor, J.W. Day, L.P. Ranum, Myotonic dystrophy type 2 caused by a CCTG expansion in intron 1 of ZNF9, Science 293 (2001) 864 867.

[3] J.W. Day, K. Ricker, J.F. Jacobsen, L.J. Rasmussen, K.A. Dick, W. Kress, C. Schneider, M.C. Koch, G.J. Beilman, A.R. Harrison, J.C. Dalton, L.P. Ranum, Myotonic dystrophy type 2: molecular, diagnostic and clinical spectrum, Neurology 60 (2003) 657-664.

[4] A. Mankodi, E. Logigian, L. Callahan, C. McClain, R. White, D. Henderson, M. Krym, C.A. Thornton, Myotonic dystrophy in transgenic mice expressing an expanded CUG repeat, Science 289 (2000) 1769-1773.

[5] J.M. Houseley, Z. Wang, G.J. Brock, J. Soloway, R. Artero, M. Perez-Alonso, K.M. O’Dell, D.G. Monckton, Myotonic dystrophy associated expanded CUG repeat muscleblind positive ribonuclear foci are not toxic to Drosophila, Hum. Mol. Genet. 14 (2005) 873 883.

[6] M. Mutsuddi, C.M. Marshall, K.A. Benzow, M.D. Koob, I. Rebay, The spinocerebellar ataxia 8 noncoding RNA causes neurodegeneration and associates with staufen in Drosophila, Curr. Biol. 14 (2004) 302-308.

[7] C. Voisine, A.C. Hart, Caenorhabditis elegans as a model system for triplet repeat diseases, Methods Mol. Biol. 277 (2004) 141-160.

[8] J.A. Parker, M. Arango, S. Abderrahmane, E. Lambert, C. Tourette, H. Catoire, C. Neri, Resveratrol rescues mutant polyglutamine cytotoxicity in nematode and mammalian neurons, Nat. Genet. 37 (2005) 349-350.

[9] H. Wang, P.J. Lim, C. Yin, M. Rieckher, B.E. Vogel, M.J. Monteiro, Suppression of polyglutamine-induced toxicity in cell and animal models of Huntington's disease by ubiquilin, Hum. Mol. Genet. 15 (2006) 1025-1041.

[10] T. Gidalevitz, A. Ben-Zvi, K.H. Ho, H.R. Brignull, R.I. Morimoto, Progressive disruption of cellular protein folding in models of polyglutamine diseases, Science 311 (2006) 1471-1474.

[11] T. Haaf, G. Sirugo, K.K. Kidd, D.C. Ward, Chromosomal localization of long trinucleotide repeats in the human genome by fluorescence in situ hybridization, Nat. Genet. 12 (1996) 183-185.

[12] C. Mello, A. Fire, DNA transformation, Methods Cell Biol. 48 (1995) 451-482.

[13] S. Brenner, The genetics of Caenorhabditis elegans, Genetics 77 (1974) 71-94.

[14] J.A. Lewis, J.T. Fleming, Basic culture methods, Methods Cell Biol. 48 (1995) 3-29.

[15] R.H. Waterston, D. Hirsh, T.R. Lane, Dominant mutations affecting muscle structure in Caenorhabditis elegans that map near the actin gene cluster, J. Mol. Biol. 180 (1984) 473-496.

[16] D.M. Raizen, L. Avery, Electrical activity and behavior in the pharynx of Caenorhabditis elegans, Neuron 12 (1994) 483-495.

[17] L. Avery, D. Raizen, S. Lockery, Electrophysiological methods, Methods Cell Biol. 48 (1995) 251-269.

[18] H. Seznec, O. Agbulut, N. Sergeant, C. Savouret, A. Ghestem, N. Tabti, J.C. Willer, L. Ourth, C. Duros, E. Brisson, C. Fouquet, G. Butler-Browne, A. Delacourte, C. Junien, G. Gourdon, Mice trans- 
genic for the human myotonic dystrophy region with expanded CTG repeats display muscular and brain abnormalities, Hum. Mol. Genet. 10 (2001) 2717-2726.

[19] P. Jin, D.C. Zarnescu, F. Zhang, C.E. Pearson, J.C. Lucchesi, K. Moses, S.T. Warren, RNA-mediated neurodegeneration caused by the fragile $\times$ premutation $\mathrm{rCGG}$ repeats in Drosophila, Neuron 39 (2003) 739-747.

[20] C.J. McLeod, L.V. O'Keefe, R.I. Richards, The pathogenic agent in Drosophila models of 'polyglutamine' diseases, Hum. Mol. Genet. 14 (2005) 1041-1048.
[21] L.T. Timchenko, J.W. Miller, N.A. Timchenko, D.R. DeVore, K.V. Datar, L. Lin, R. Roberts, C.T. Caskey, M.S. Swanson, Identification of a (CUG) $n$ triplet repeat RNA-binding protein and its expression in myotonic dystrophy, Nucleic Acids Res. 24 (1996) 4407-4414.

[22] C.A. Milne, J. Hodgkin, ETR-1, a homologue of a protein linked to myotonic dystrophy, is essential for muscle development in Caenorhabditis elegans, Curr. Biol. 9 (1999) 1243-1246.

[23] M. Fujita, T. Kawano, A. Ohta, H. Sakamoto, Neuronal expression of a Caenorhabditis elegans elav-like gene and the effect of its ectopic expression, Biochem. Biophys. Res. Commun. 260 (1999) 646-652. 